\title{
Internal color gradients and the color-magnitude relation of early-type galaxies
}

\author{
Marco Scodeggio \\ Istituto di Fisica Cosmica "G. Occhialini”, via Bassini 15, I-20133, Milano, Italy \\ (marcos@ifctr.mi.cnr.it)
}

\begin{abstract}
The traditional use of fixed apertures in determining the well known colormagnitude (CM) relation of early type galaxies, coupled with the presence of radial color gradients within these systems, introduces a bias in the CM relation itself. The effect of this bias is studied here deriving a CM relation which is based on color measurements carried out homogeneously within an aperture of radius equal to that of the galaxy effective radius. A sample of 48 giant early-type galaxies in the Coma cluster, with CCD observations in the $U$ - and $V$-band, is used for this derivation. It is found that internal radial color gradients in early-type galaxies cannot be neglected when discussing the colors of these systems, and that the CM relation derived using color measurements within the effective radius is significantly flatter than those based on fixed-aperture color measurements. With the presently available data it is impossible to determine whether the relation is completely flat, or whether a small correlation is still present between galaxy color and luminosity.
\end{abstract}

Subject headings: galaxies: elliptical and lenticular, cD - galaxies: fundamental parameters - galaxies: stellar content

\section{Introduction}

It has long been known that the colors of early-type galaxies correlate with their absolute magnitude (e.g. Baum 1959; de Vaucouleurs 1961; McClure \& van den Bergh 1968; Lasker 1970). This color-magnitude (CM) relation was demonstrated to be a universal property of early-type galaxies by Visvanathan \& Sandage (1978), and has become one of the most commonly used tools to constrain the epoch of formation and the star formation history of these systems. Bower, Lucey \& Ellis (1992a,b; hereafter BLE92) were the first to study the CM relation using photometric data based on CCD observations, which exhibited an even smaller scatter around the mean relation than previous determinations. More recently the CM relation has been shown to be present in clusters up to $z \sim 1$ (e.g. Stanford, Eisenhardt \& Dickinson 1998; Kodama et al. 1998a; Gladders et al. 1998), and to have similar properties in distant and nearby clusters.

These observations, when interpreted within the framework of a rapid dissipative galactic collapse (e.g. Larson 1974), and with the help of synthetic stellar population models (e.g. Buzzoni 
1989; Bruzual \& Charlot 1993; Worthey 1994; Tantalo et al. 1996; Kodama \& Arimoto 1997), yield an estimate for the epoch of early-type galaxy formation of $z \sim 4.5$, with a fairly limited star formation activity taking place afterwards (Bower, Kodama \& Terlevich 1998). The systematic change of color as a function of luminosity in these models is interpreted as the result of systematic changes of the mean metallicity as a function of galaxy mass. These results currently provide what is probably the strongest argument against the otherwise quite successful models of hierarchical galaxy formation (but see Kauffmann \& Charlot 1998 for a different point of view).

The seemingly straightforward exercise of deriving a CM relation observationally is complicated by the presence of systematic radial color gradients within giant early-type galaxies. It is now well established that these objects are reddest in their cores, and become bluer towards their peripheries (e.g. de Vaucoleurs 1961; Sandage \& Visvanathan 1978; Frogel et al. 1978; Franx, Illingworth \& Heckman 1989; Peletier et al. 1990a; Peletier, Valentijn \& Jameson 1990b), most likely as a result of radial metallicity gradients within their stellar populations (see recent analysis by Tamura et al. 2000, and Saglia et al. 2000). Since the galaxy colors used in almost all studies of the CM relation are measured within apertures of fixed radius, different portions of a galaxy are used to derive these colors, depending on the galaxy intrinsic size (and therefore on its luminosity). As a result, a spurious correlation between galaxy luminosity and color is produced, even in the absence of systematic changes of the global stellar population as a function of galaxy luminosity. This problem has been recognized for quite some time, and a number of attempts have been made to take it into consideration. For example Sandage \& Visvanathan (1978) corrected their color measurements to a constant fraction of each galaxy isophotal radius $(\theta / D(0)=0.5)$, but this correction does not solve the problem, since isophotal radii include a variable fraction of galaxy light, depending on galaxy luminosity. Other correction schemes have been attempted on the modeling side, for synthetic stellar population models (e.g. Kodama et al. 1998a; Kauffmann \& Charlot 1998). In this case mean radial color gradient values are used to convert model-based total magnitudes into aperture ones, for a direct comparison with the observations. However, given the large scatter that is observed for the intensity of radial color gradients (see for example Peletier et al. 1990a; or section 4 , it is not appropriate to use a mean value to characterize this property of the galaxy population. It would be clearly preferable to derive observationally the CM relation using variable-size apertures, measuring galaxy colors at a fixed fraction of the galaxy light.

In this work a sample of 48 early-type galaxies in the Coma cluster, with photometric observations in the $U$ - and $V$-band and measurements of the galaxy effective radius in the $V$ and $I$-band, all available from the literature, is used to derive a CM relation that is based on color measurements within the effective radius for each galaxy in the sample. This newly obtained relation should be directly comparable to the model predictions based on synthetic stellar population models. The data-set used in this work is described in section 2; the derivation of the traditional "fixed-aperture" and of the new "fixed light fraction" CM relation are described in section 3; color gradients within individual galaxies are discussed in section 4 ; section 5 presents the discussion and the conclusions of this work. 


\section{The data}

A sample of early-type galaxies in the Coma cluster was chosen for this work, for a number of practical and astrophysical reasons. The Coma cluster is among the richest nearby clusters, and has therefore a large population of early-type galaxies. Due to its proximity, accurate morphological information and a large set of photometric and spectroscopic observations are available. Being a rich cluster, it represents also a fair local counterpart to the distant clusters being currently used to study the processes of formation and evolution of early-type galaxies. The Coma cluster is however too distant to allow an expansion of the study of the CM relation to dwarf galaxies. This exercise will be described in a future work (Scodeggio et al. 2001), based on a sample of early-type galaxies in the Virgo cluster.

The dataset being used here consists of $U$ - and $V$-band CCD photometry observations of E and S0 galaxies in the Coma cluster, taken from BLE92, supplemented by determinations of each galaxy effective radius $r_{e}$ (the radius within which is contained half of the galaxy light) taken from Lucey et al. (1991) and from Scodeggio, Giovanelli \& Haynes (1998; hereafter SGH98). BLE92 obtained observations for 81 galaxies at the $2.5 \mathrm{~m}$ Isaac Newton Telescope (INT), in La Palma. For each galaxy these authors provide a series of aperture magnitude measurements, carried out within synthetic apertures with radius ranging from 4 to 60 arcsec. The typical accuracy of these measurements is 0.03 mag. Of these 81 objects, 56 were observed both in the $U$ - and in the $V$-band, and they are those used here. Total galaxy magnitudes in the $V$-band, $V^{T}$, are also provided by BLE92, who re-calibrated the original data from Godwin \& Peach (1977).

Effective radius measurements for a subset of these galaxies were presented by Lucey et al. (1991), based in part on independent $V$-band photometric observations also carried out at the INT, and in part on BLE92 $V$-band data. The effective radius was measured at the "half light" point of the growth curve of each galaxy. Unfortunately, only 21 galaxies of the 56 with $U$ - and $V$-band photometry are in the Lucey et al. sample. A much larger fraction of these are in the I-band sample presented by SGH98, based on CCD photometry observations carried out with the $0.9 \mathrm{~m}$ telescope of the Kitt Peak National Observatory. These $r_{e}$ measurements are based on $r^{1 / 4}$ profile fits (see SGH98 for details), that were extended from an inner radius twice as large as that of the seeing disk to an outer radius chosen to match the radial extent of the galaxy spheroidal component: for $\mathrm{E}$ galaxies this radius is the one of the outermost reliable isophote, while for S0 galaxies it is the radius where the disk component begins to dominate the galaxy surface brightness. Independent measurements for a number of these galaxies were presented also by Jørgensen, Franx \& Kjærgaard (1995), and by Saglia et al. (1997). The statistical uncertainty in the determination of $r_{e}$ is approximately $6 \%$, but significantly larger systematic uncertainties could be produced by the particular fitting method used to derive $r_{e}$ and the galaxy's effective surface brightness, leading to differences that, on a given object, can be as large as $50 \%$ (see, for example, the discussion in SGH98). While a complete discussion on the reliability of effective radius measurements is beyond the scope of the present work, it is interesting to verify the possibility of using the larger set of $I$-band $r_{e}$ measurements instead of the smaller set of $V$-band 
ones, despite the significant wavelength difference. As there is considerable overlap between the Lucey et al. and the SGH98 sample, it is possible to make a detailed comparison between the two. The two sets of $r_{e}$ measurements appear to be in rather good agreement, as shown in Figure 1, where the fractional difference $\Delta r_{e} / r_{e}$ is plotted as a function of both $r_{e}$ and the galaxy total magnitude, for the 55 galaxies that are common to the Lucey et al. and the SGH98 sample (this overlap includes galaxies that are not in the BLE92 sample used here). The average fractional difference is 0.092 , with an rms scatter of 0.183 , but a non-zero difference could be expected, as a consequence of having used different photometric bands and different methodologies to carry out the measurements. The important finding is that the differences between $r_{e}$ measured in different bands do not depend on galaxy luminosity or size, and therefore using $r_{e}$ as measured in the $I$-band, as will be done hereafter (unless specifically stated) does not introduce any luminosity or color related bias in the following analysis.

For each galaxy, aperture magnitudes at $r_{e}$ were derived independently in the two bands interpolating (or using a very small extrapolation where necessary) the relative growth curve data. Given the good sampling of each galaxy growth curves, and the fact that for most galaxies the virtual aperture with radius $r_{e}$ is tightly bracketed by a pair of aperture measurements, simple linear relations between aperture magnitude and the logarithm of the aperture radius were used for the interpolation/extrapolation. Only 8 of the 48 objects in the sample required an extrapolation of the measurements to derive aperture magnitudes at $r_{e}$. In all cases this was an extrapolation to a radius smaller than the smallest aperture radius used for the measurements $(4$ $\operatorname{arcsec})$. This procedure was preferred to the intuitively simpler one of just dividing by two the total flux measured for a given galaxy (which would be equivalent to defining $m\left(r_{e}\right)=m_{T}+0.753$ ), because the latter always requires a relatively large extrapolations to be carried out on the measurements to derive total fluxes. This extrapolation process would undoubtedly become the largest source of color measurement uncertainty, while the procedure adopted here only adds to the original photometric uncertainty the uncertainty on the determination of $r_{e}$, which is significantly smaller (see section 3.2). Galaxy colors within $r_{e}$ were obtained taking the difference between the relative aperture magnitudes. As a consistency check colors were also obtained by interpolating/extrapolating a linear relation between color and the logarithm of the aperture radius. Differences between the two techniques show an rms scatter of 0.018 mag, when only interpolation between observations is required to derive the color within $r_{e}$. Only colors derived from the difference of aperture magnitudes are used in the following analysis.

Among the 55 objects with $r_{e}$ measurements both in $V$ - and $I$-band, 20 are also in the BLE92 sample, and for them it was possible to obtain a color measurement within apertures of radius $r_{e}$ as determined in the two bands. The differences between these two measurements are shown in Figure 2, as a function of galaxy magnitude, effective radius, and color. As could be expected from the above discussion on the differences between effective radii measured in the two bands, no significant difference is present between the two sets of color measurements, except for a small offset introduced by the somewhat larger values of $r_{e}$ as determined in the $I$-band. The 
average difference between the $U-V$ colors is $-0.018 \mathrm{mag}$, with an $r m s$ scatter of 0.032 mag. The measured scatter is in good agreement with the expected uncertainty in the color measurements introduced by measurement errors on $r_{e}$ (see also Section 3.2). The fact that the color difference is independent from galaxy luminosity and color means that no bias is introduced in the $[(U-V), V]$ $\mathrm{CM}$ relation by the choice of measuring colors within an aperture of radius the $I$-band effective radius.

The sample used in the following analysis is therefore composed of 48 galaxies with magnitude measurements both in $U$ - and $V$-band, and a measurement of $r_{e}$ in the $I$-band. Morphological types for all objects are available from Dressler (1980): 23 of them are classified as Ellipticals, and 25 as S0. The sample is limited to galaxies brighter than $V \simeq 15.8$, but does not obey any strict completeness criterion. Assuming a distance modulus for Coma of 34.75, the limiting magnitude corresponds to $M_{V} \simeq-19.0$, or equivalently $M_{B} \simeq-18.0$.

\section{The Color-Magnitude relation}

\subsection{The standard "fixed-aperture" relation}

The standard "fixed-aperture" CM relation was derived using two slightly different approaches. First, the relation was obtained in the form that is most often used in the analysis of high-redshift clusters, i.e. using both color and magnitude measurements within a given fixed aperture (e.g. Bower et al. 1998; Kodama, Bower \& Bell 1998b; Gladders et al. 1998). In the second approach, which was introduced to facilitate the comparison with the "fixed light fraction" relation discussed in Section 3.2, colors measured in fixed apertures were correlated with magnitudes measured within the aperture of radius $r_{e}$ and with total magnitudes. This approach is very similar to the one used by BLE92 and Stanford et al. (1998).

The two procedures produce very similar results, except for the fact that using a fixed aperture both for the color and the magnitude measurement introduces the bias related to sampling different portions of a galaxy depending on its luminosity (and therefore also on its color) in both quantities, and this makes the observed CM relation steeper. This is illustrated in Figure 3, where the $[(U-V), V]$ CM relation obtained when measuring the colors within an aperture of 10 arcsec, and the magnitudes within the same 10 arcsec aperture, within $r_{e}$, or when using total magnitudes, are compared. The slope obtained when using total magnitudes is $-0.074 \pm 0.008$, in good agreement with the one previously reported by BLE92 $(-0.082 \pm 0.008)$.

One should also notice that the slope of the "fixed-aperture" CM relation is marginally related to the size of the fixed aperture used for the color measurements, becoming slightly flatter for larger aperture radii. This flattening, although not statistically significant in the present sample, is in good agreement with a similar result reported by Okamura et al. (1999), based on $B-R$ color measurements also in the Coma cluster. 
Table 1 presents a summary of the linear least square fits to the CM relations discussed in this work. All fits are direct fits, where the fitting procedure minimizes the vertical distances between data-points and the best-fit line, taking into account only error measurements in the independent variable, the color in this case. This fitting procedure was adopted because for the total magnitude measurements individual uncertainty estimates are not available, and also because the main goal of this paper is to illustrate the relative changes in the CM relation when different methods are used to derive it, more than to derive the ultimate value of the relation parameters. It is certainly possible that such a very simplistic approach to the fitting of the CM relation might bias the result towards flatter slopes, especially when measurements errors are large. This might be happening within the present dataset, as shown by the comparison between the correlation involving magnitudes measured within a 10 arcsec aperture (first line in the Table; accurate measurements) and the correlation involving total magnitudes or magnitudes within $r_{e}$ (second and third line in the Table; less accurate measurements). Uncertainties in the value of the best-fit slope and zero point were obtained with the statistical jackknife method. With a sample of $N$ data-points, the fit is repeated $N$ times using $N-1$ points, each time excluding a different point, to derive an estimate of the parent population from which the parameter under examination is derived. With the present dataset, formal uncertainties on the best-fit slopes are approximately one order of magnitude smaller than those based on the statistical jackknife. However, one must keep in mind that the two given uncertainties are highly correlated, and cannot be used independently to asses the global uncertainty affecting the fit. An approximate estimate of the zero point uncertainty component that is statistically independent from the slope uncertainty is given by the accuracy with which one can measure the average value of the residuals from the best-fit line, which is typically of 0.02 mag. The scatter in the CM relation reported in the last column of the table is the rms scatter of the differences between the measured color and the one predicted by the fit.

\subsection{The relation at a fixed galaxy light fraction}

As discussed in Section 1, if there are radial color gradients within galaxies, the use of fixed apertures for color measurements introduces a bias in the CM relation, as more luminous galaxies have larger $r_{e}$ (see, for example, Fish 1964; Guzmán, Lucey \& Bower 1993; Pahre, Djorgovski $\&$ de Carvalho 1998), and therefore larger overall extent. Since radial color and line strength gradients are commonly present within giant early-type galaxies (see, for example, de Vaucoleurs 1961; Sandage \& Visvanathan 1978; Frogel et al. 1978; Franx et al. 1989; Peletier et al. 1990a,b on the color gradients, and Couture \& Hardy 1988; Gorgas, Efstathiou \& Aragón-Salamanca 1990; Davies, Sadler \& Peletier 1993; Carollo \& Danziger 1994 on the line strength ones) one can expect

the "fixed-aperture" CM relation discussed in the previous section to provide a biased view of the color properties of early-type galaxies.

To quantify the relevance of this effect, a "fixed light fraction" CM relation was derived, 
using the colors measured within each galaxy effective radius. With this procedure, always a fixed fraction of the galaxy light contributes to the color measurements, allowing a more meaningful comparison between galaxies of different luminosities. Contrary to what appeared to be the implicit assumption in most previous works on the CM relation, the bias introduced by the use of fixed apertures appears to be significant. Figure 4 shows a comparison of the CM relations obtained when using color measurements within a fixed aperture of $10 \operatorname{arcsec}$, and within $r_{e}$. It is clear that any correlation between galaxy color and luminosity largely disappears when colors are measured within each galaxy effective radius. The slope of the best fitting linear relation between color and magnitude goes from $-0.074 \pm 0.008$ (as measured for the fixed aperture relation) to $-0.016 \pm 0.018$, which is statistically compatible with a completely flat relation. A similarly shallow slope for the CM relation derived measuring galaxy colors homogeneously at the effective radius was previously reported by Prugniel \& Simien (1996) and by van Dokkum et al. (1998), although those authors do not comment specifically on the significance of this point.

Some concern about the reality of this result might be raised by the fact that color measurements within $r_{e}$ are affected by larger uncertainties than those within a fixed radius aperture, because of the uncertainty in the determination of $r_{e}$ itself. As discussed in Section 2, the statistical uncertainty in the determination of $r_{e}$ for the present sample is approximately $6 \%$ (SGH98), but much larger systematic uncertainties could be present, introduced by the specific fitting procedure used to derive the value of $r_{e}$ from a galaxy surface brightness profile (see, for example, Figures 4 to 6 in SGH98). Therefore a total uncertainty of approximately 20-30\% might be a better estimate of the error budget involved in the determination of $r_{e}$. However, the impact on the present color measurements of a conservative $20 \% r_{e}$ uncertainty estimate (the scatter between $V$ - and $I$-band measurements discussed in Section 2 would lead to an estimate of approximately 10-12\%) is only $\pm 0.025 \mathrm{mag}$, on average. Since this uncertainty is always smaller than, or comparable to the intrinsic uncertainty of the presently available photometric measurements, it does not have a significant impact on the results presented here. The 0.03 mag uncertainty quoted by BLE92 for their magnitude measurements translates into a 0.042 mag uncertainty in the fixed-aperture color determinations, and the addition of the contribution from $r_{e}$ determination uncertainties brings the total fixed galaxy fraction color uncertainty to approximately $0.05 \mathrm{mag}$. These uncertainties are reported in Figure 4 , together with a somewhat arbitrary estimate of 0.15 mag for the uncertainty in the total magnitude measurements.

\section{Radial color gradients}

The significant changes in the CM relation properties described in the previous section point towards the relevance of internal color and stellar population gradients within early-type galaxies for a detailed understanding of their properties and evolutionary histories. The average color gradient $d(U-V) / d(\log r)$ measured in the sample used here is of -0.15 mag per dex in

radius, with an rms scatter around this mean value of 0.15 mag per dex in radius. This is in 
very good agreement with the findings of Peletier et al. (1990a), who report an average gradient of $-0.16 \pm 0.11 \mathrm{mag}$ per dex in radius. Note that the use, in both cases, of a simple power-law method to measure the color gradients makes the comparison meaningful, although different methods are used to measure the color points.

In agreement with previous studies, no significant correlation is found between the strength of the color gradient and the galaxy luminosity (see Figure 5). There is however a marginal indication that the scatter in gradient strength might be smaller for the brighter objects in our sample. This result is somewhat in contrast with the claim presented by Peletier (1993) that early-type galaxies fainter than $\mathrm{M}_{B}=-19.5$ have significantly smaller color gradients than those observed in more luminous galaxies. Unfortunately the number of bright galaxies in the present sample is too small to allow a clear determination of this effect.

The rather large scatter that is observed globally for the values of the color gradient is responsible for the larger scatter measured in the fixed galaxy fraction version of the CM relation with respect to the fixed aperture version. This fact points towards a very high photometric accuracy requirement for future measurements aimed at measuring with good accuracy the slope of the CM relation. Also, it becomes quite clear that using an average gradient value to correct color measurements derived from either observations or theoretical models is not appropriate. In fact the scatter in gradient values observed among real galaxies is comparable in magnitude with the mean value itself, instead of representing a small additional uncertainty superposed to the main effect one is considering. Therefore when using a mean gradient value for the color corrections one would neglect the effects of the relatively strong gradients that are present in a significant fraction of the galaxy population.

\section{Discussion and Conclusions}

The results presented above show that internal radial color gradients in early-type galaxies cannot be neglected when discussing the colors of these systems. The strength of the gradients measured here is in agreement with other measurements previously reported in the literature, although differences in samples and in the photometric bands used by different authors make a detailed comparison difficult. It is confirmed that for giant early-type galaxies the strength of the color gradient is not correlated with the galaxy luminosity. Also, a fairly large scatter in this strength is observed at all luminosities, although there is an indication in the present dataset that this scatter might be smaller for the brightest objects.

One point of concern with the present dataset is the rather heterogeneous nature of the observations and data reduction procedures adopted to derive magnitudes and effective radii. However one should note that a substantial flattening of the CM relation when colors are measured consistently at the galaxies effective radius, is obtained also when considering $I$ - and $H$-band data, with effective radii measured homogeneously within the same data set (Scodeggio et al. 2001). 
Very shallow "fixed light fraction" CM relation slopes have been published also by Prugniel \& Simien (1996) and van Dokkum et al. (1998).

While it is found that colors measured within the effective radius depend less strongly on the galaxy luminosity than previously believed, it is not possible to accurately determine whether a small correlation is still present between the two quantities, or whether giant E and S0 galaxies all have the same color, within a small scatter. Because only small differences in color are involved, a larger, more homogeneous dataset, comprising data of higher photometric accuracy than those used here, will be required to settle this point. In any case the present result implies that some modification of the models of galaxy formation currently discussed in the literature is necessary. The shallower slope (if not the complete flatness) of the CM relation, and the consequential somewhat larger scatter allowed in the colors of early-type galaxies with respect to that measured in the fixed-aperture relation, should significantly relax some of the tight constraints so far taken into account in discussing the allowed star formation histories of these systems and the processes that led to their formation.

The present results apply only to giant E and S0 galaxies, since with this dataset one can sample only the bright end of the early-type galaxies luminosity function (spanning an interval of approximately five magnitudes). The picture appears to become more complex when one considers also dwarf systems. We are currently extending our analysis to the Virgo cluster, to build a sample that includes also dE and dS0 galaxies (Scodeggio et al. 2000). Our preliminary finding is that a global blueing trend with decreasing luminosity is present among early-type galaxies. The trend is significant for the global sample of Virgo cluster galaxies, spanning an interval of more than ten magnitudes in luminosity, but becomes vanishingly small at the bright end of the distribution, in agreement with the results presented here. This would suggest the possibility of a CM relation which is divided into two different regimes, one for the giant and one for the dwarf galaxies.

It would be quite natural to extend the conclusions reached here to other well known scaling relations for early-type galaxies, like the metallicity-luminosity relation, exemplified primarily by the $\mathrm{Mg}_{2}-\sigma$ relation (e.g. Bender, Burstein \& Faber 1993). In this case once again the measurements are made using a fixed aperture. The typical size of the aperture is comparable with the galaxy $r_{e}$ for the small, low luminosity and low $\sigma$ galaxies, while it is one order of magnitude smaller than $r_{e}$ in the case of the large, luminous, high $\sigma$ ones. Since there are radial gradients in the strength of the $\mathrm{Mg}_{2}$ index, the approach may introduce a significant bias in the inferred mass-metallicity relation. However a recent analysis of a relatively large set of measurements of such gradients has led Kobayashi \& Arimoto (1999) to conclude that a metallicity-mass relation is present among elliptical galaxies even when mean metallicities are considered.

The conclusion is that the interpretation of the CM relation of bright early-type galaxies, as well as that of similar relations with galaxy luminosity that might suffer from the same "fixed-aperture plus internal gradient" bias, might need to be revised. This could lead to a significant change in the overall observational picture of early-type galaxies. In particular it 
could no longer be required to have a very strong correlation between galaxy luminosity (which is approximately equivalent to mass) and mean metallicity, allowing for a more significant

contribution from random events (like mergers, or a number of star formation episodes besides the original burst) in the formation and evolutionary processes that shape these objects.

The most sincere thanks to Alessandro Boselli, Peppo Gavazzi, Riccardo Giovanelli, and Martha Haynes for their contributions to this project, and to Stephane Charlot, Mark Dickinson, Alvio Renzini, Piero Rosati, and Roberto Saglia for useful discussions on the subject.

\section{REFERENCES}

Baum, W.A., 1959, PASP, 71, 106

Bender, R., Burstein, D., Faber, S.M., 1993, ApJ, 411, 153

Bower, R.G., Lucey, J.R., Ellis, R.S., 1992a, MNRAS, 254, 589 (BLE92)

Bower, R.G., Lucey, J.R., Ellis, R.S., 1992b, MNRAS, 254, 601 (BLE92)

Bower, R.G., Kodama, T., Terlevich, A., 1998, MNRAS, 299, 1193

Bruzual, A.G., Charlot, S., 1993, ApJ, 405, 538

Buzzoni, A., 1989, ApJS, 71, 817

Carollo, C.M., Danziger, I.J., 1994, MNRAS, 270, 523

Couture, J., Hardy, E., 1988, AJ, 96, 867

Davies, R.L., Sadler, E.M., Peletier, R.F., 1993, MNRAS, 262, 650

de Vaucouleurs, G., 1961, ApJS, 5, 233

Dressler, A., 1980, ApJS, 42, 565

Fish, R.A., 1964, ApJ, 139, 284

Franx, M., Illingworth, G.D., Heckman, T., 1989, AJ, 98, 538

Frogel, J.A., Persson, S.E., Aaronson, M., Matthews, K., 1978, ApJ, 220, 75

Gladders, M.D., Lopez-Cruz, O., Yee, H.K.C., Kodama, T., 1998, ApJ, 501, 571

Godwin, J.G., \& Peach, J.V., 1977, MNRAS, 181, 323

Gorgas, J., Efstathiou, G., Aragón-Salamanca, A., 1990, MNRAS, 245, 217 
Guzmán, R., Lucey, J.R., Bower, R.G., 1993, MNRAS, 265, 731

Kauffmann, G., Charlot, S., 1998, MNRAS, 294, 705

Kobayashi, C., Arimoto, N., 1999, ApJ, 527, 573

Kodama, T., Arimoto, N., 1997, A\&A, 320, 41

Kodama, T., Arimoto, N., Barger, A.J., Aragón-Salamanca, A., 1998a, A\&A, 334, 99

Kodama, T., Bower, R.G., Bell, E.F., 1998b, astro-ph/9810138

Jørgensen, I., Franx, M. \& Kjærgaard, P. 1995, MNRAS, 273, 1097

Larson, R.B., 1974, MNRAS, 166, 585

Lasker, B.M., 1970, AJ, 75, 21

Lucey, J.R., Guzmán, R., Carter, D., Terlevich, R.J., 1991, MNRAS, 253, 584

McClure, R.D., van den Bergh, S., 1968, AJ, 73, 1008

Okamura, S., et al., 1999, in "Cosmological Parameters and the Evolution of the Universe", ed. Sato, K., (Kluwer: Dordrecht), p. 160

Pahre, M.A., Djorgovski, S.G., de Carvalho, R.R., 1998, AJ, 116, 1591

Peletier, R.F., 1993, in "Structure, Dynamics, and Chemical Evolution of Elliptical Galaxies", ed. Danziger, I.J., Zeilinger, W.W., Kjaer, K., (ESO: Garching) p. 409

Peletier, R.F., Davies, R.L., Illingworth, G.D., Davis, L.E., Cawson, M., 1990a, AJ, 100, 1091

Peletier, R.F., Valentijn, E.A., Jameson, R.F., 1990b, A\&A, 233, 62

Prugniel, P., Simien, F., 1996, A\&A, 309, 759

Saglia, R.P., et al., 1997, MNRAS, 292, 499

Saglia, R.P., Maraston, C., Greggio, L., Bender, R., Ziegler, B., 2000, A\&A, 360, 911

Sandage, A., Visvanathan, N., 1978, ApJ, 223, 707

Scodeggio, M., Giovanelli, R., Haynes, M.P., 1998, AJ, 116, 2728 (SGH98)

Scodeggio, M., et al., 2001, in preparation

Stanford, S.A., Eisenhardt, P.R., Dickinson, M., 1998, ApJ, 492, 461

Tamura, N., Kobayashi, C., Arimoto, N., Kodama, T., Ohta, K., 2000, AJ, 119, 2134 
Tantalo, R., Chiosi, C., Bressan, A., Fagotto, F., 1996, A\&A, 311, 361

van Dokkum, P.G., Franx, M., Kelson, D.D., Illingworth, G.D., Fisher, D., Fabricant, D., 1998, ApJ, 500, 714

Visvanathan, N., Sandage, A., 1977, ApJ, 216, 214

Worthey, G., 1994, ApJS, 95, 107 

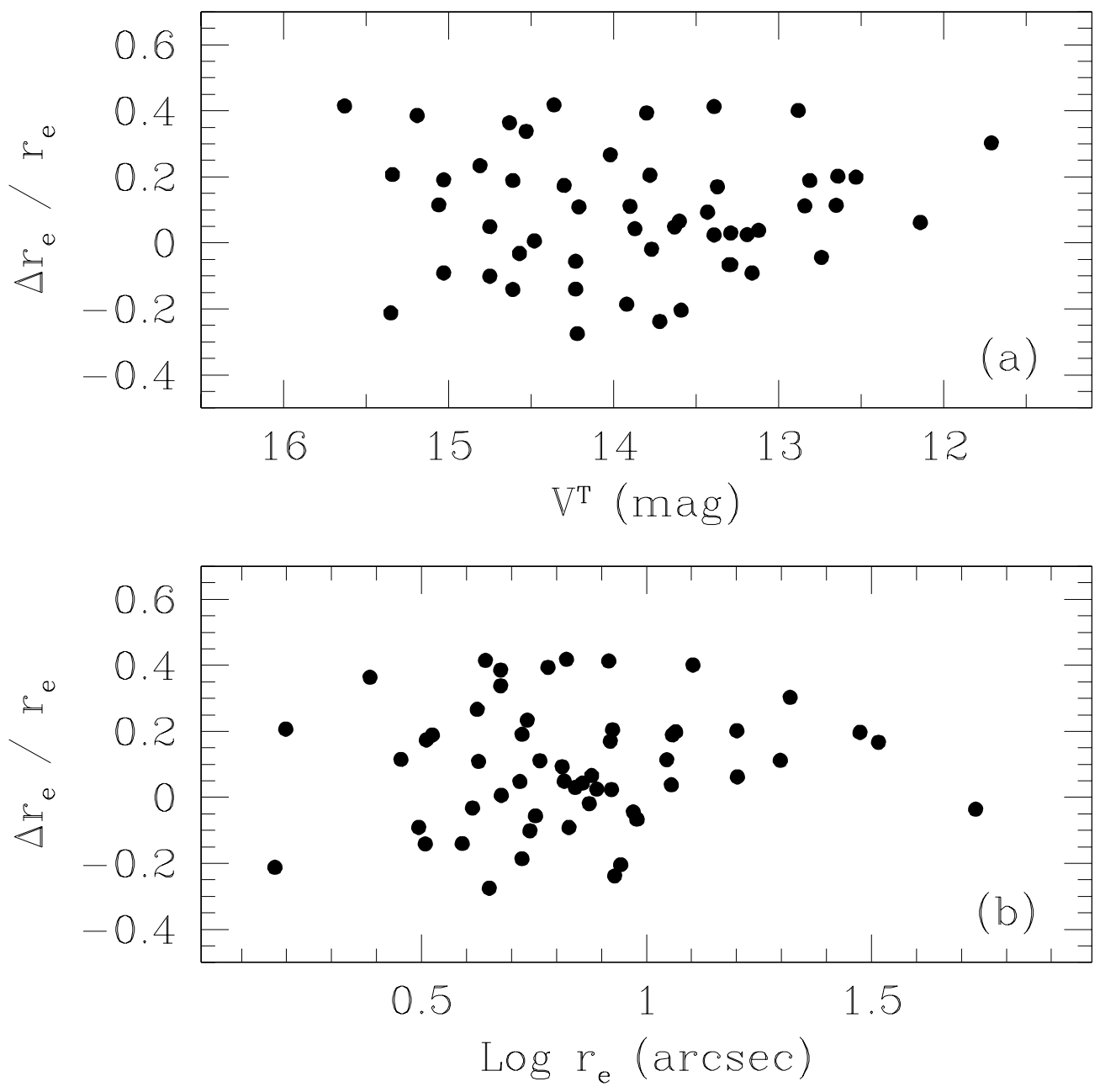

Fig. 1.- Differences in the values of effective radius as measured in the $V$ - (Lucey et al. 1991) and $I$-band (Scodeggio, Giovanelli \& Haynes 1998). In the two panels the ratio of the difference between the $r_{e}$ measurements in the $I$ - and $V$-band with respect to their mean is plotted as a function of the galaxy total magniude (panel a) or the logarithm of the $I$-band measurement (panel b). 

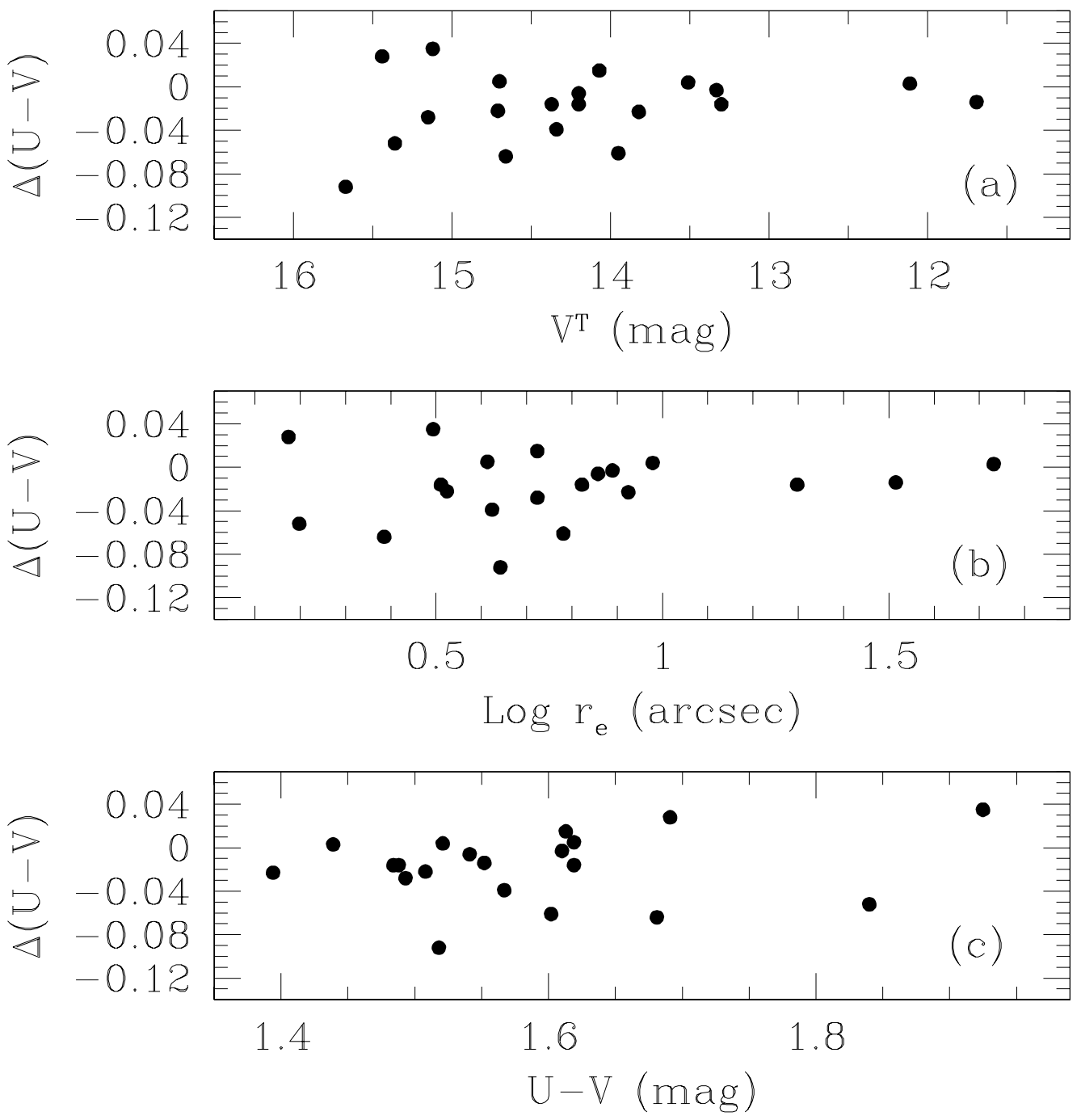

Fig. 2.- Differences in the values of the $U-V$ color, as measured within an aperture of radius the $V$ - and $I$-band effective radius. In the three panels the difference between the color measured within the $I$-band and the $V$-band $r_{e}$ is plotted as a function of the galaxy total magnitude (panel a), the logarithm of the $I$-band effective radius (panel b), and the $U-V$ color measured within that radius (panel c). 

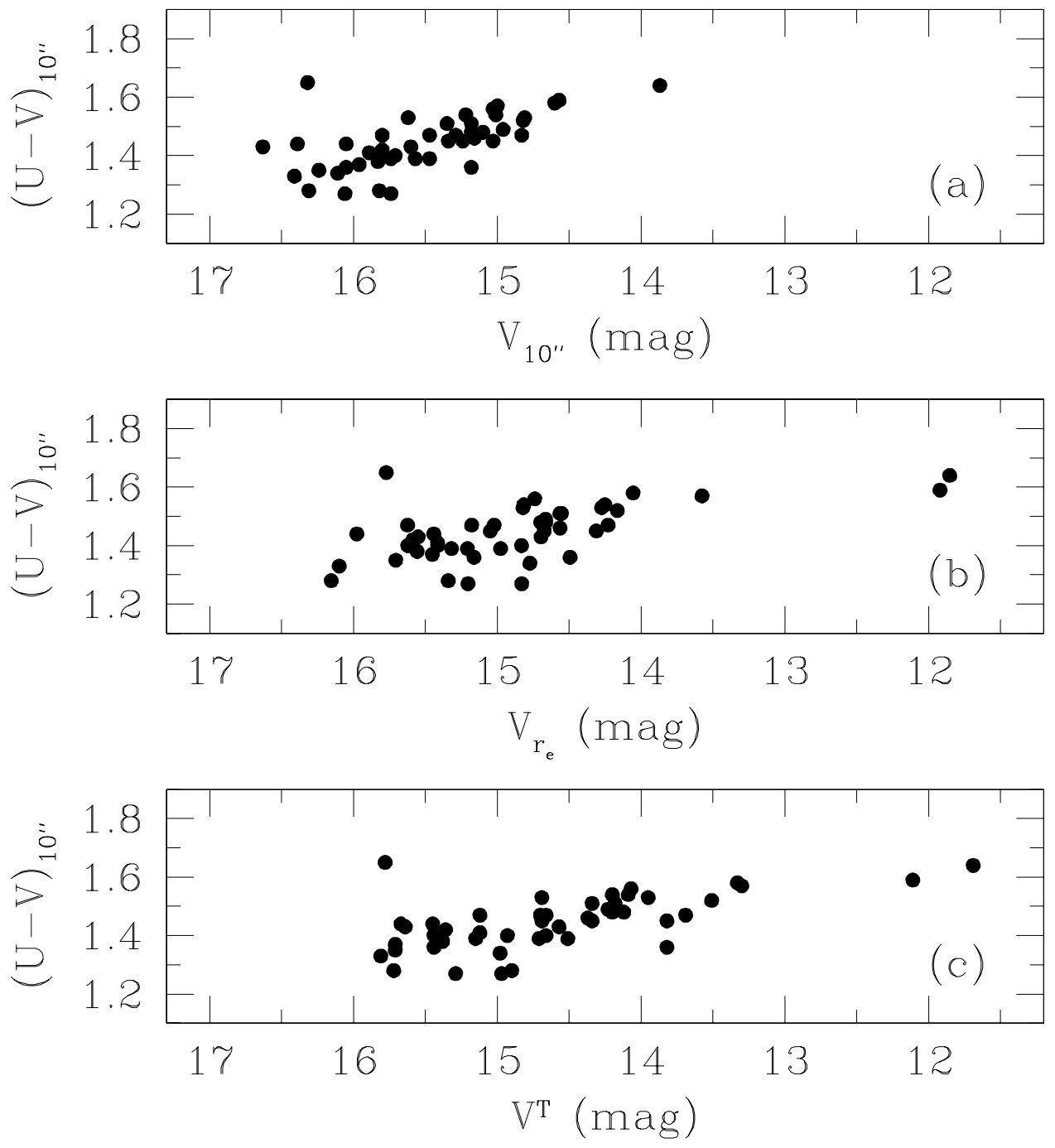

Fig. 3.- "Fixed-aperture" color-magnitude relations. The $[(U-V), V]$ relation is plotted in three different formats: in panel (a) both color and magnitude are measured within a 10 arcsec aperture; in panels (b) and (c) the color is the same as in panel (a), but the magnitudes are the one measured within an aperture of radius the galaxy effective radius (panel b) and the total magnitude (panel c). 


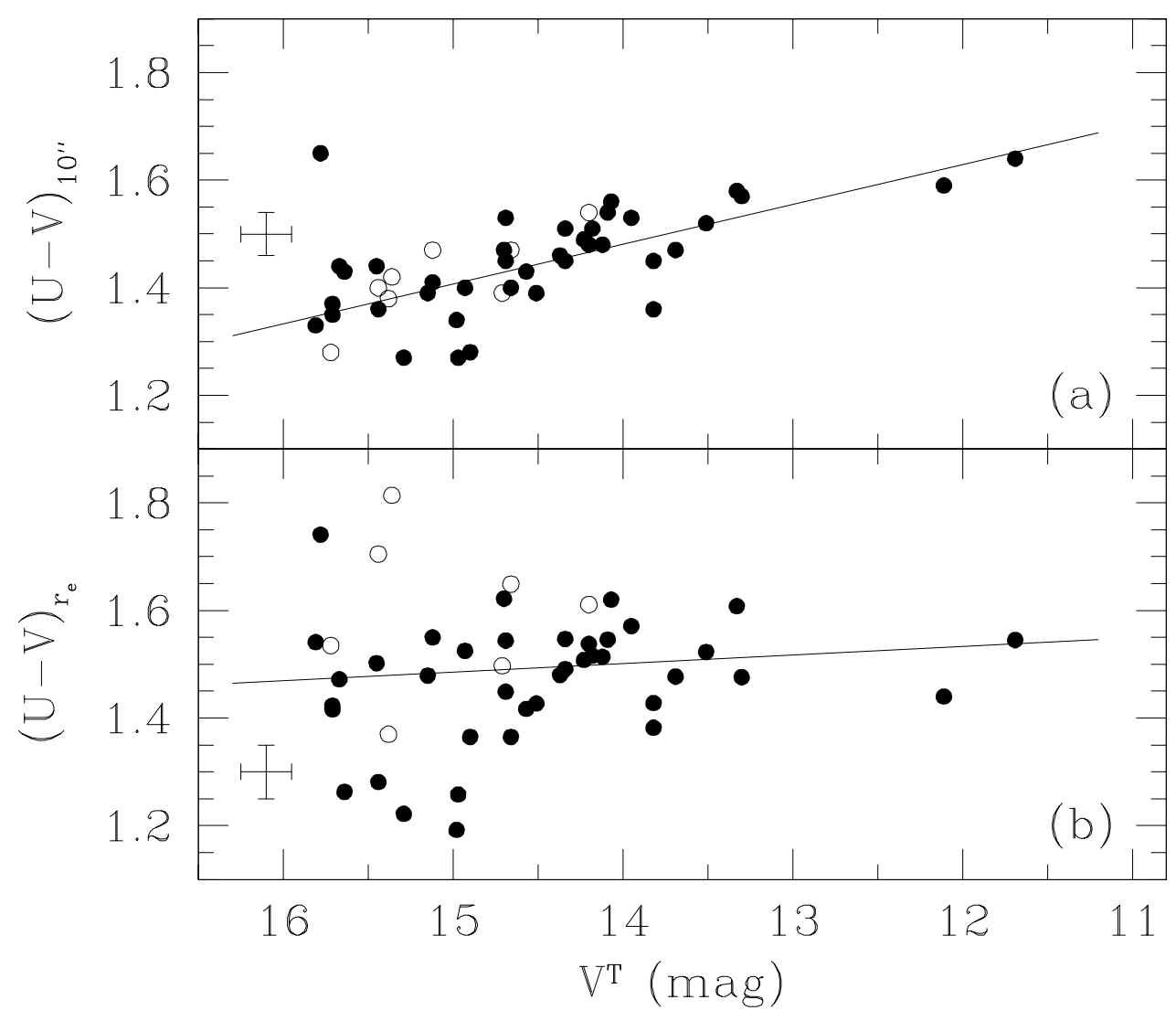

Fig. 4.- Comparison between "fixed-aperture" and "fixed light fraction" color-magnitude relations. Panel (a) refers to the color measured within a fixed 10 arcsec aperture, while the panel (b) refers to the color measured within the galaxy effective radius. In both cases the magnitude is the galaxy total magnitude. Empty symbols identify those galaxies that required an extrapolation of their growth curve to derive the color measurement at the effective radius. The solid lines represent the best fit to the relation discussed in the text. The "crosses" identify typical uncertainties in the plotted colors and magnitudes. 

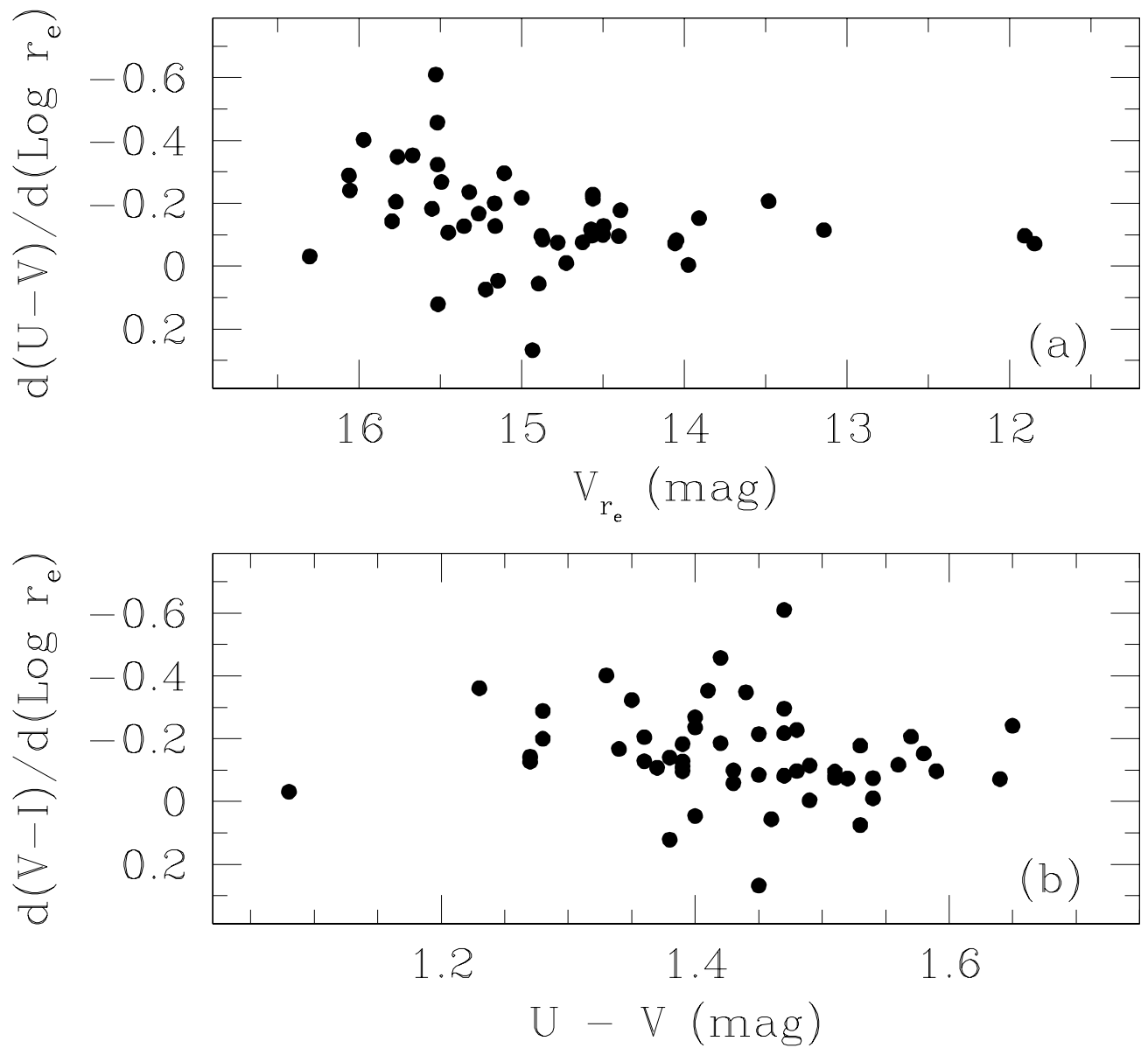

Fig. 5. - The strength of the radial internal color gradient $d(U-V) / d \log (r)$, as a function of galaxy luminosity (represented here by the integrated magnitude within the galaxy effective radius; panel a), and galaxy color (panel b). 
Table 1. Derived $[(U-V), V]$ color-magnitude relations

\begin{tabular}{cccc}
\hline \hline Apertures $^{\mathrm{a}}$ & slope & zero point & scatter (mag) \\
\hline $10^{\prime \prime}, 10^{\prime \prime}$ & $-0.119 \pm 0.026$ & $3.28 \pm 0.40$ & 0.080 \\
$10^{\prime \prime}, r_{e}$ & $-0.072 \pm 0.012$ & $2.50 \pm 0.21$ & 0.086 \\
$10^{\prime \prime}$, tot. & $-0.074 \pm 0.008$ & $2.52 \pm 0.11$ & 0.059 \\
$r_{e}$, tot. & $-0.016 \pm 0.018$ & $1.73 \pm 0.28$ & 0.136 \\
\hline
\end{tabular}

athe format for this column is (aperture used for the color measurement),(aperture used for the magnitude measurement). 